Bio - grafía. Escritos sobre la Biología y su Enseñanza. ISSN 2027-1034

Edición Extraordinaria. p.p. 1548 - 1555

Memorias del IX Encuentro Nacional de Experiencias en Enseñanza de la Biología y la Educación Ambiental. IV Congreso Nacional de Investigación en Enseñanza de la Biología.

\title{
APRENDIZAJE PROFESIONAL A PARTIR DE LAESTRATEGIA METODOLÓGICA PARA EL DESARROLLO: RINDE CON-CIENCIA
}

\author{
Eduardo Ravanal Moreno
}

\section{RESUMEN}

Reflexionar sobre las acciones de enseñanza contribuye al aprendizaje del profesor, y esto a su vez, a la mejora de la práctica de enseñanza. Reconociendo aquello, es necesario implementar estrategias de desarrollo que contribuyan a dinamizar y vincular el pensamiento del profesor con su práctica pedagógica y vice versa. En ese marco, se diseña la estrategia metodológica RINDE con-Ciencia. Ésta surge de un proyecto de investigación con profesores de biología. La estrategia distingue 12 etapas, a partir de las cuales, se pretende ayudar al profesor a reflexionar (R), interpretar (IN) y decidir (DE) usando el conocimiento profesional de manera consciente (Con-Ciencia) para reconceptualizar su enseñanza en pos de mejores aprendizajes.

PALABRAS CLAVE: aprendizaje profesional, estrategia metodológica, desarrollo profesional

\begin{abstract}
ABST ACT
Reflecting on teaching actions contributes to the teacher's learning, and this in turn, to improving teaching practice. Recognizing this, it is necessary to implement development strategies that contribute to dynamize and link the teacher's thinking with their pedagogical practice and vice versa. Within this framework, the methodological strategy RINDE conCiencia is designed. The strategy distinguishes 12 stages, from which it is intended to help the teacher to reflect $(R)$, interpret $(I N)$ and decide $(D E)$ using professional knowledge consciously (Con-Science) to reconceptualize their teaching in pursuit of better learning.
\end{abstract}

KEYWORD: professional learning, methodological strategy, professional development

\section{INTRODUCCIÓN}

El desarrollo profesional es un proceso que si logra ser efectivo, puede influir en el aprendizaje de los profesores (Kang, Cha y Ha, 2013). Sin embargo, las iniciativas de desarrollo profesional, en general, son desagregadas y desconectados de los problemas de la práctica de enseñanza (Zhang, Parker, Koehler y Eberhardt, 2015) demostrando por

1 Centro de estudios e Investigación. Universidad Central de Chile. Iravanalm@ucentral.cl 
Bio - grafía. Escritos sobre la Biología y su Enseñanza. ISSN 2027-1034

Edición Extraordinaria. p.p. 1548 - 1555

Memorias del IX Encuentro Nacional de Experiencias en Enseñanza de la Biología y la

Educación Ambiental. IV Congreso Nacional de Investigación en Enseñanza de la Biología.

ello, una baja efectividad en el aprendizaje del profesor. Por eso que hoy, propuestas innovadoras focalizadas en el crecimiento del profesor, son necesarias y valoradas. San Antonio et al., (2011) plantean que el desarrollo profesional contribuye a profundizar el conocimiento del profesor y el desarrollo de las habilidades de enseñanza, ambos, necesarios para monitorear el progreso de los estudiantes, por eso, que el desarrollo profesional según Zhang et al., (2013) debe estar sustentado en una teoría de aprendizaje, ser intenso y sostenido, focalizado hacia un contenido, brindar oportunidades para activar y enriquecer el aprendizaje y, conectarse con la práctica diaria del profesor.

Si el desarrollo profesional docente es clave para mejorar el conocimiento y la práctica de enseñanza del profesor (Kang et al., 2013), entonces es importante investigar y proponer estrategias de desarrollo innovadoras para dichos propósitos, como pretende ser la estrategia RINDE con-Ciencia.

Considerando la complejidad de la enseñanza y del pensamiento del profesor (Porlán \& Rivero, 1998), pensamos en tres andamios de desarrollo para ayudar al profesor a reflexionar sobre su quehacer profesional, que son: Preocupaciones, Dificultades y Problemas de enseñanza. En ese marco, las preocupaciones profesionales representan la vía de pensamiento espontáneo e inicial sobre el cual el profesorado inicia su reflexión. En tanto, los problemas representan el constructo más abstracto para el profesor y de mayor demanda cognitiva, dado que su abordaje, implica un reflexión profunda que permita reconocer y decidir qué conocimiento usar y para qué tipo de acciones de enseñanza según la demanda del problema de enseñanza. En esa complejidad, surge la estrategia metodológica para el desarrollo, RINDE con-Ciencia cuyo objetivo es promover la reflexión individual y entre pares considerando los estadios de desarrollo profesional de Kugel (1993). RINDE con-Ciencia es la Reflexión, Interpretación y Decisión del profesor usando el conocimiento de manera consciente en pos de enriquecer su práctica de enseñanza.

\section{ESTRATEGIA METODOLÓGICA RINDE CON-CIENCIA}

La enseñanza exige al profesor, inevitablemente, tomar decisiones y, en lo posible buenas decisiones, en tiempos breves y espacios complejos, cuestión que nos lleva a preguntarnos: ¿qué y cómo hacer consciente al profesor para que comprenda sus acciones ampliamente, distinguiendo en ellas, las potencialidades y limitaciones que tienen para el aprendizaje de sus estudiantes? Su abordaje desde dos marcos teóricos: el modelo intercomunicado de Clarke y Hollinsworth (2002) y los aportes de la visión profesional (Sherin, Jacobs, y Philipp, 2011). Surge entonces RINDE con-Ciencia.

\section{ETAPAS DE LA ESTRATEGIA RINDE CON-CIENCIA}

\section{Etapa 0. Situaciones contextuales y situadas del quehacer del profesor}

La experiencia recogida nos indica que el aprendizaje del profesor debe partir de situaciones contextuales y situadas. Por ello, se diseñan situaciones representativas del quehacer del profesor (el caso) para iniciar la reflexión sobre su acción y la toma de 
Bio - grafía. Escritos sobre la Biología y su Enseñanza. ISSN 2027-1034

Edición Extraordinaria. p.p. 1548 - 1555

Memorias del IX Encuentro Nacional de Experiencias en Enseñanza de la Biología y la

Educación Ambiental. IV Congreso Nacional de Investigación en Enseñanza de la Biología.

consciencia. Las situaciones $(n=60)$ derivan, inicialmente, de los encuentros con profesores y se formalizan a partir de los descriptores propuestos del dominio A y $\mathrm{C}$ del Marco para la Buena Enseñanza (Mineduc, 2008). El caso describe una situación profesional en primera persona con el objeto de garantizar el involucramiento del profesor en la actividad que cierra con una pregunta directriz que persigue la explicitación de las preocupaciones del profesor.

\section{Etapa 1. Explicitación de la(s) preocupación(es) profesionales.}

El profesor aborda la siguiente pregunta: ¿qué de lo que hiciste o de lo planteado por... te preocupa a la hora de enseñar? Se busca que el profesor explicite todo aquello que le resulte preocupante de la situación. La(s) preocupación(es) profesionales son planteadas como frases, teniendo al final de esta etapa un conjunto de preocupaciones profesionales según el contenido del caso.

\section{Etapa 2. Clasificación de la(s) preocupación(es) profesionales según énfasis}

Kugel (1993) ha descrito para la enseñanza tres planos de desarrollo, que son: profesor, contenido y estudiantes. Cada plano describe acciones de preocupación que permiten identificar un plano de desarrollo, por lo tanto, esta etapa constituye en sí mismo, un instrumento de identificación -relativa- del desarrollo del profesor. La estrategia pretende que el profesor-participante reconozca en qué plano se encuentran sus preocupaciones y decida por cuál de ellas desea comenzar su análisis.

Etapa 3. Identificar acciones de enseñanza para enfrentar la(s) preocupación(es) profesionales

La decisión y selección de la preocupación profesional, es el insumo para la explicitación de acciones docentes de enseñanza que permiten enfrentar la preocupación profesional. Para ayudar al profesor a esa tarea, se usa la siguiente pregunta mediadora: ¿qué acciones de enseñanza realiza (ría) para enfrentar la preocupación profesional descrita? Las acciones de enseñanza planteadas son analizadas, inicialmente, desde la perspectiva del aprendizaje de los estudiantes.

\section{Etapa 4. Potencialidades y limitaciones de las acciones de enseñanza para el aprendizaje}

Esta etapa consiste en el análisis de las acciones de enseñanza propuestas desde dos perspectivas, que son: acciones de enseñanza favorecedoras del aprendizaje de los estudiantes (acción dinamizadora) y acciones de enseñanza limitadoras del aprendizaje de los estudiantes (acción obstaculizadora), esto último relevante en la estrategia, dado que todas aquellas acciones, que se reconozcan, de manera consciente, como limitadores del aprendizaje, representarían una dificultad de enseñanza (Ravanal, 2017).

\section{Etapa 5. Generando Familia de dificultades para identificar un problema de enseñanza}

Las dificultades de enseñanza identificadas se agrupan según los atributos que los profesores asignen. Ahora bien, para ampliar el análisis los profesores ellos acceden a los 
Bio - grafía. Escritos sobre la Biología y su Enseñanza. ISSN 2027-1034

Edición Extraordinaria. p.p. 1548 - 1555

Memorias del IX Encuentro Nacional de Experiencias en Enseñanza de la Biología y la

Educación Ambiental. IV Congreso Nacional de Investigación en Enseñanza de la Biología.

descriptores de los factores de efectividad de enseñanza (Creemer, et al., 2013) entendidos en la estrategia como potenciales problemas.

\section{Etapa 6. Identificación de un Problema de Enseñanza}

Asumiendo que un problema de enseñanza es una situación específica al que convergen dificultades de enseñanza (una familia). Por lo tanto, su solución no es inmediata y exige del profesor el uso de un conocimiento integrado para resolverlo. Para ayudar a delimitar un problema de enseñanza, se usan algunos de los descriptores de efectividad propuestos por Creemer et al., (2013), que por la experiencia que se ha recogido, focaliza en los siguientes: Orientación, Cuestionamiento, Estructuración y Modelamiento de la enseñanza. Para favorecer su identificación, se presenta una matriz con los descriptores de cada problema (factor) para que el profesor identifique su problema (tabla 1).

Etapa 7. Externalización del conocimiento profesional disponible para abordar el problema de enseñanza

A partir de la identificación (explicitación del problema de enseñanza) se busca que el profesor identifique el conocimiento que le permite reconocer los atributos de una situación particular de enseñanza (Hennisen et al., 2017) para decidir y actuar. La etapa 7 pretende que el profesor reconozca qué del conocimiento profesional domina y puede ser usado para abordar el problema identificado.

\section{Etapa 8. Explicitar el conocimiento profesional necesario para abordar el problema de enseñanza}

Junto con externalizar el conocimiento disponible para abordar un problema de enseñanza específico, es importante también que el profesor reconozca el conocimiento necesario para abordar un problema de enseñanza. Particularmente esta etapa, pretende dos cosas: otorgar valor a las fuentes o referentes teóricos para el profesor y reconocer la importancia del conocimiento didáctico como aquél necesario para enseñar, y por tanto clave, para enfrentar un problema de enseñanza.

Etapa 9. Explicación de las acciones de enseñanza y sus consecuencias para la enseñanza

En la etapa 4 de la estrategia el profesor-participante identifica potencialidades y limitaciones de algunas acciones docentes para el aprendizaje. En esta ocasión, se busca que el profesor vuelva a analizar las acciones de enseñanza propuesta, pero, desde la perspectiva de la enseñanza. En ese sentido, ocurren dos cosas, se invita al profesorparticipante a reflexionar sobre el modo en qué se implementaría la acción docente, a través de la pregunta ¿por qué hacerlo así? -explíquelo-, en tanto y como segundo punto, el profesor-participante debe identificar las consecuencias de esas acciones para la enseñanza que pretende promover y, que de alguna forma, sería distinta para abandonar la preocupación profesional inicial. 
Bio - grafía. Escritos sobre la Biología y su Enseñanza. ISSN 2027-1034

Edición Extraordinaria. p.p. 1548 - 1555

Memorias del IX Encuentro Nacional de Experiencias en Enseñanza de la Biología y la Educación Ambiental. IV Congreso Nacional de Investigación en Enseñanza de la Biología.

Tabla 1. Atributos de un factor clave de enseñanza o problema de enseñanza

\begin{tabular}{|l|l|}
\hline $\begin{array}{l}\text { Problemas } \\
\text { Enseñanza }\end{array}$ & Atributos paradigmáticos \\
\hline Orientación & $\begin{array}{l}\text { - Realizo acciones docentes que ayuden a los estudiantes a } \\
\text { comprender el objetivo de aprendizaje de la clase. }\end{array}$ \\
- Realizo acciones docentes que ayuden a los estudiantes a \\
comprender los objetivos de todas las actividades y tareas \\
de aprendizaje que se realizan en la clase. \\
- Realizo acciones docentes que ayuden a los estudiantes a \\
identificar la(s) razón(es) que hay detrás de las actividades y \\
tareas que se proponen.
\end{tabular}


Bio - grafía. Escritos sobre la Biología y su Enseñanza. ISSN 2027-1034

Edición Extraordinaria. p.p. 1548 - 1555

Memorias del IX Encuentro Nacional de Experiencias en Enseñanza de la Biología y la

Educación Ambiental. IV Congreso Nacional de Investigación en Enseñanza de la Biología.

\section{Etapa 10. Análisis de consecuencias -positivas/negativas- pensando en la venseñanza y el aprendizaje}

El análisis de la acción de enseñanza desde la perspectiva del profesor y su rol, supone que toda acción docente tiene consecuencias para acciones docentes ulteriores, por ello, la etapa 10 pretende que el profesor-participante identifique y visibilice todas aquellas consecuencias (positivas o negativas) para la enseñanza que arrastra una acción y, que inicialmente, constituyen una solución a la preocupación profesional inicial. El objetivo es analizar la acción del profesor desde la perspectiva del problema de enseñanza identificado. Estos aspectos y resultados, son la base para comenzar a decidir que dejar de hacer en clase en pos de una mejor enseñanza.

Etapa 11. Decidir sobre las acciones para mejorar la práctica de enseñanza: qué mantengo; qué debo cambiar.

La identificación de una preocupación profesional y las acciones de enseñanza que pueden ayudar a dejar de lado una intranquilidad profesional derivan de la reflexión del profesor (R). Alertar sobre las consecuencias de una acción como solución ante una preocupación es, a su vez, una oportunidad para evaluar su efecto en el aprendizaje de los estudiantes -ampliación de las perspectivas del profesor-. Por ello, el análisis de las acciones docentes desde ambos planos, contribuyen a mejorar la capacidad para interpretar (IN) una situación específica que alberga un problema de enseñanza para decidir (DE). Consecuencia de ello, el profesor vuelve al espacio de reflexión para decidir, personal y conscientemente, qué acciones cambiar o mantener para mejorar la práctica de enseñanza.

\section{Etapa 12. Compromiso profesional}

Siendo el trabajo individual y colaborativo muy relevante para el aprendizaje del profesor, creemos que el sentido profesional de equipo capaz de enfrentar la enseñanza y sus complejidades debe culminar con una instancia que fortalezca el trabajo realizado, ya sea sistematizando lo realizado y reconociendo además, el aporte que cada miembro hace al conocimiento del profesor. Surge el compromiso profesional, como una instancia de síntesis de ideas y de trabajo en equipo. 
Bio - grafía. Escritos sobre la Biología y su Enseñanza. ISSN 2027-1034

Edición Extraordinaria. p.p. 1548 - 1555

Memorias del IX Encuentro Nacional de Experiencias en Enseñanza de la Biología y la Educación Ambiental. IV Congreso Nacional de Investigación en Enseñanza de la Biología.

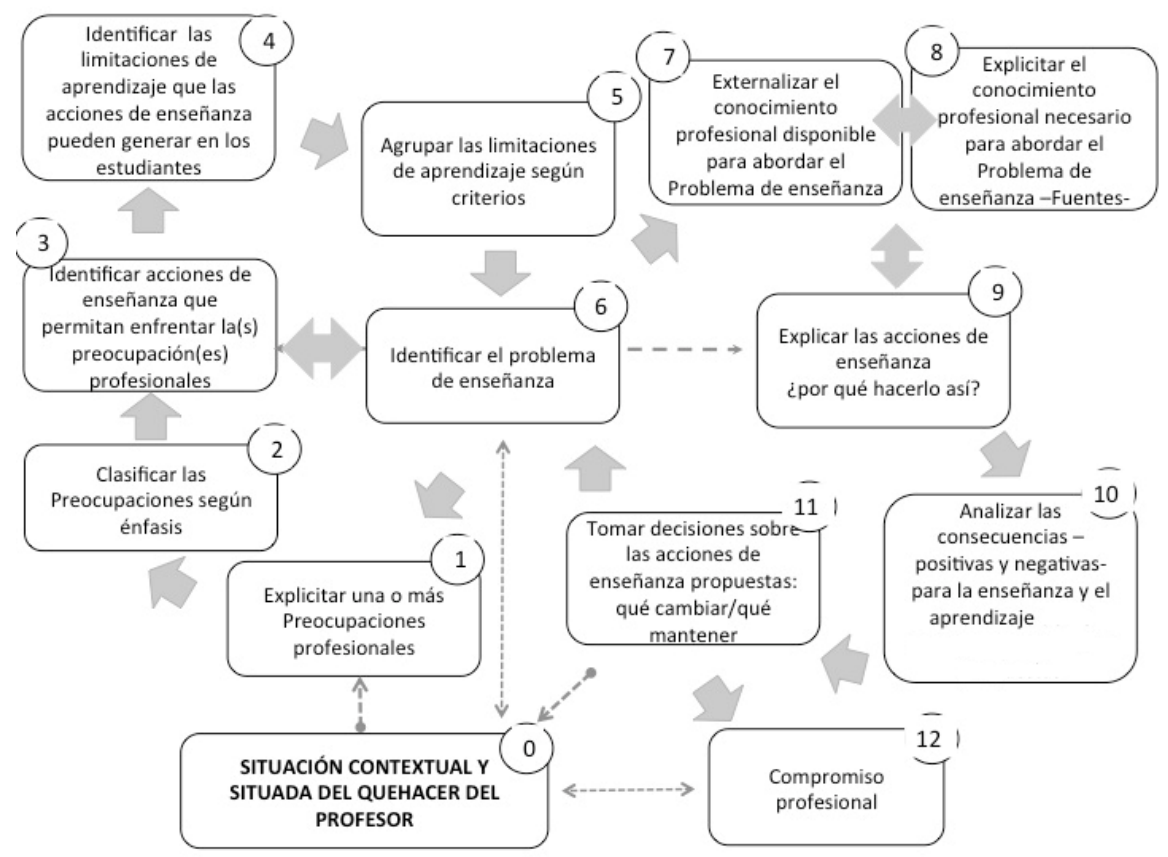

Figura 1. Etapas de la estrategia RINDE con-Ciencias

\section{BIBLIOGRAFÍA}

Clarke, D. \& Hollingsworth, H. (2002). Elaborating a model of teacher professional growth. Teaching and Teacher Education, 18(8), 947-967

Hennissen, P., Beckers, H. y Moerkerke, G. (2017). Linking practice to theory in teacher education: A growth in cognitive. Teaching and Teacher Education, 63, 314-325.

Kang, H., Cha, J. y Ha, B. (2013). What Should We Consider in Teachers Professional Development Impact Studies? Based on the Conceptual Framework of Desimone. Creative Education, 4 (4A), 11-18. Zhang, Parker, Koehler y Eberhardt, 2015

Kugel, P. (1993). How professors develop as teachers. Studies in Higher Education, 18 (3), 315-328.

Ministerio de Educación, Mineduc (2008). Marco para la Buena Enseñanza. Santiago: Centro de Perfeccionamiento, Experimentación e Investigación Pedagógica.

Porlán, R. \& Rivero, A. (1998). El conocimiento de los profesores. España: Diada. 
Bio - grafía. Escritos sobre la Biología y su Enseñanza. ISSN 2027-1034

Edición Extraordinaria. p.p. 1548 - 1555

Memorias del IX Encuentro Nacional de Experiencias en Enseñanza de la Biología y la Educación Ambiental. IV Congreso Nacional de Investigación en Enseñanza de la Biología.

Ravanal, E. (2017). Consideraciones para un programa de desarrollo profesional que oriente al profesor a reconceptualizar su enseñanza. Revista Científica, 1(8), 60-71.

Creemer, B., Kyriakides, L. y Antoniou, P. (2013). Teacher professional development for improving quality of teaching. Dordrecht, the Netherlands: Springer.

San Antonio, D.; Morales, N. y Moral, L. (2011). Module-based professional development for teachers: a cost-effective Philippine experiment. Teacher Development, 15(2), 157 169.

Sherin, M., Jacobs, V. y Philipp, R. (Eds.) (2011). Mathematics teacher noticing: seeing through teachers 'eyes. Ney York: Routledge. 